\title{
Effects of Organic and Inorganic Fertilizers on the Growth of NH-Ae 47-4 Variety of Okra
}

\section{MOLIK A. ZAINAB, ELUWA C. VINCENT, OLUWATOBI S. AYODELE*, LAKWANNUM, GAYUS Y. AND OLORUNMAIYE S. KEHINDE}

\author{
Department of Plant Biology, Faculty of Life Sciences, University of Ilorin, PMB 1515, Ilorin, Nigeria. \\ Corresponding author: ayodeleoluwatobi@ gmail.com ${ }^{*},+2347064987030^{*}$ \\ ksolorunmaiye@yahoo.com
}

\begin{abstract}
The paper evaluates the effects of organic and inorganic fertilizers on the growth of okra (variety NH-Ae 47-4. Organic fertilizers (cow dung and poultry droppings) and inorganic (NPK 15:15:15 and Urea 64:0) fertilizers were used for the experiment. The fertilizers were weighed and applied at $5 \mathrm{~g}, 10 \mathrm{~g}$ and $15 \mathrm{~g}$ and were replicated three times and each having a control. Vegetative growth parameters taken include, shoot fresh weight (g), dry weight $(\mathrm{g})$, plant height $(\mathrm{cm})$, leaf number, stem girth $(\mathrm{cm})$, leaf area $\left(\mathrm{cm}^{2}\right)$. The results obtained from the experiment showed that the effect of the treatments were significantly difference from the control for all the parameters accessed with urea fertilizer having least effect. Plants treated with poultry litters have best performance by recording the highest fresh and dry weight $(0.39 \mathrm{~g})$ at 4 weeks after planting (WAP); highest stem height $29.33 \mathrm{~cm}$ for all the concentrations applied. Similarly, it has highest leaf area and stem girth $\left(64.67 \mathrm{~cm}^{2}\right.$ and $2.23 \mathrm{~cm}$ respectively) at $8 \mathrm{WAP}$. $\odot J A S E M$ http://dx.doi.org/10.4314/jasem.v20i1.24
\end{abstract}

Keywords: evaluate, cow-dung, poultry-droppings, okra, vegetative.

\section{Introduction}

Okra (Abelmoschus esculentus Moench) is an annual, herbaceous flowering plant in the Mallow family that originated from tropical and subtropical Africa and is natural to the West Africa (Aladele et al., 2008). Okra is mainly cultivated for its young immature fruits and consumed as a vegetable, raw, cooked or fried in countries like Sudan, Egypt and Nigeria; it is also important in other tropical areas including Asia central and South America often used as ingredient of soups and sauces. The fruits can be conserved by drying or pickling, the roasted seed is considered as coffee substitute; the leaves, flower buds, flowers and calyces can be eaten cooked as greens (Ajari et al., 2003). In Nigeria, okra is grown in both wet and dry season but attract a larger profit in the dry season when the demand is often in excess with limited supplies (Ayeni et al., 2012). Okra is a good source of vitamins, minerals, calories and amino acid found in seeds and compares favorably with those in poultry, eggs and soybean (Brady and Weil, 1999).

Okra cultivation requires nutrients such as nitrogen $(\mathrm{N})$, phosphorus $(\mathrm{P})$, potassium $(\mathrm{K})$, calcium $(\mathrm{Ca})$, sodium (Na) and Sulphur (S) for fertility maintenance and crop production. These nutrients are specific in function and must be supplied to plants at the right time and at the right quantity. Lack of sufficient amounts of these nutrients result in poor performance of the okra with growth been affected resulting to low yield (Chauhan, 1972). In developing countries like Nigeria, the population growth rate is so high that improved technologies including rational use of fertilizers must be employed to meet the food requirement of the people (Hedrick, 1972). Improving soil fertility through the application of fertilizers is an essential factor enabling the world to feed the billions of people that are added to its population (Hera, 1996). Declining soil fertility is a major production constraint in Africa, especially in Nigeria, and it is becoming increasingly critical to secure sustainable soil productivity (Massomo and Rweyemamu, 1989; Oladotun, 2002). Organic manure helps to improve the physical condition of soil and provide adequate amount of necessary nutrients for the soil productivity (Qhureshi, 2007).

Thus there is a need for cultivation of okra with increased yield and quality, which this present study is aimed at investigating which of the following fertilizer application; organic (Poultry litter, cow dung) and inorganic (NPK 15:15:15: and Urea 64:0) fertilizer will be more effective on the growth and yield of okra (Abelmoschus esculentus).

\section{MATERIALS AND METHOD}

The study was carried out between January 2014April 2014 at the Agricultural Research site of the faculty of Agriculture in the University of Ilorin, Ilorin Nigeria. The variety of okra (Abelmoschus 
esculentus Moench) used was $\mathrm{NH}$ Ae 47-4. The materials used in carrying out this experimental work includes; Cow dung, poultry litter, NPK (15: 15: 15), Urea fertilizer (46: 0: 0), petri-dishes and spatula, watering balance, hoe, thread, potting buckets, hand shovel, sandy-loamy topsoil, meterrule, tape rule, foil paper, oven and writing materials.

Forty-eight plastic buckets (five-litre capacity each), viable seeds of $\mathrm{NH}$ Ae 47-4 varieties of okra, inorganic fertilizers (NPK 15:15:15 and Urea 46:0:0), organic fertilizers (cow dung and poultry litters) were used for the experiment.

Each of the buckets was perforated and filled with sandy-loam soil. Each of the fertilizers weighing $5 \mathrm{~g}$, $10 \mathrm{~g}$ and $15 \mathrm{~g}$ replicated three times was mixed with the soil in the buckets each having a control (i.e. without fertilizer). The cow dung and poultry litter was ground into a powdery form and sieved prior to weighing and mixing with the soil. The fertilizers were mixed 3 days prior to sowing the seeds.

Germination ranged between 4 to 7 days and germination count was taken after a week. Ample watering was done to avoid rotting of the seedlings. Seeds were re-sown in buckets which had no germination. Measurement of growth parameters commenced 3 weeks after planting with careful hand weeding to avoid pest and nutrient competition. Data were collected 3 weeks after planting (WAP) and at weekly intervals thereafter. The growth parameters at the vegetative stage taken on a weekly basis include; shoot fresh weight $(\mathrm{g})$, dry weight $(\mathrm{g})$, plant height $(\mathrm{cm})$, leaf number, stem girth $(\mathrm{cm})$, leaf area $\left(\mathrm{cm}^{2}\right)$.

Data were subjected to Analysis of Variance (ANOVA). Significantly different mean values were separated, using the Duncan's Multiple Range Test (DMRT) at 5\% significant level.

\section{RESULTS AND DISCUSSION}

Poultry litter, Cow dung and NPK fertilizers showed significant effect on the growth of the variety of okra used in the experiment while urea fertilizer had an exceptionally poor effect on the growth of the variety of okra.

The fresh and dry weights of $\mathrm{NH}$ Ae 47-4 variety of okra in NPK 15:15:15 (5g) treated soil were significantly higher at 3 weeks after planting (WAP) than the other treatments. The fresh and dry weights of the okra variety in poultry litter $(10 \mathrm{~g}$ and $15 \mathrm{~g}$ ) treated soil were higher than the other treatments at 3 and 4 WAP. Urea-grown okra had the least weight for both fresh and dry weights as shown on Table 1 and 2.

Table1: Effects of Organic (Cow dung and poultry litter) and Inorganic (NPK and Urea) fertilizers on fresh and dry shoot weight of NH-Ae 47-4

\begin{tabular}{|c|c|c|c|c|c|c|c|c|c|c|c|c|c|c|c|c|}
\hline \multirow{3}{*}{$\begin{array}{c}\text { TREATMENT } \\
\text { (g) }\end{array}$} & \multicolumn{4}{|c|}{ Cow dung } & \multicolumn{4}{|c|}{ Poultry litter } & \multicolumn{4}{|c|}{ NPK } & \multicolumn{4}{|c|}{ Urea } \\
\hline & \multicolumn{2}{|c|}{$\begin{array}{c}\text { Fresh Weight (FW) } \\
\text { Week After } \\
\text { Planting(WAP) }\end{array}$} & \multicolumn{2}{|c|}{$\begin{array}{c}\text { Dry Weight (DW) } \\
\text { Week After } \\
\text { Planting(WAP) }\end{array}$} & \multicolumn{2}{|c|}{$\begin{array}{c}\text { Fresh Weight (FW) } \\
\text { Week After } \\
\text { Planting(WAP) }\end{array}$} & \multicolumn{2}{|c|}{$\begin{array}{l}\text { Dry Weight (DW) } \\
\text { Week After } \\
\text { Planting(WAP) }\end{array}$} & \multicolumn{2}{|c|}{$\begin{array}{c}\text { Fresh Weight (FW) } \\
\text { Week After } \\
\text { Planting(WAP) }\end{array}$} & \multicolumn{2}{|c|}{$\begin{array}{l}\text { Dry Weight (DW) } \\
\text { Week After } \\
\text { Planting(WAP) }\end{array}$} & \multicolumn{2}{|c|}{$\begin{array}{c}\text { Fresh Weight (FW) } \\
\text { Week After } \\
\text { Planting(WAP) }\end{array}$} & \multicolumn{2}{|c|}{$\begin{array}{c}\text { Dry Weight (DW) } \\
\text { Week After } \\
\text { Planting(WAP) }\end{array}$} \\
\hline & 3 & 4 & 3 & 4 & 3 & 4 & 3 & 4 & 3 & 4 & 3 & 4 & 3 & 4 & 3 & 4 \\
\hline Control & $0.66^{\mathrm{a}}$ & $1.04^{\mathrm{a}}$ & $0.13^{\mathrm{a}}$ & $0.23^{\mathrm{a}}$ & $0.43^{\mathrm{a}}$ & $0.50^{\mathrm{a}}$ & $0.11^{\mathrm{a}}$ & $0.07^{\mathrm{a}}$ & $0.30^{\mathrm{a}}$ & $0.00^{\mathrm{a}}$ & $0.10^{\mathrm{a}}$ & $0.00^{\mathrm{a}}$ & $0.64^{\mathrm{a}}$ & $0.31^{\mathrm{a}}$ & $0.12^{\mathrm{a}}$ & $0.09^{\mathrm{a}}$ \\
\hline 5 & $1.35^{\mathrm{a}}$ & $1.48^{\mathrm{a}}$ & $0.26^{\mathrm{a}}$ & $0.26^{\mathrm{a}}$ & $0.86^{\mathrm{a}}$ & $2.40^{\mathrm{a}}$ & $0.16^{\mathrm{b}}$ & $0.48^{\mathrm{a}}$ & $1.44^{\mathrm{a}}$ & $3.52^{\mathrm{a}}$ & $0.20^{\mathrm{a}}$ & $0.48^{\mathrm{a}}$ & $0.69^{\mathrm{a}}$ & $1.41^{\mathrm{b}}$ & $0.14^{\mathrm{b}}$ & $0.35^{\mathrm{a}}$ \\
\hline 10 & $0.83^{\mathrm{a}}$ & $1.58^{\mathrm{a}}$ & $0.19^{\mathrm{a}}$ & $0.29^{\mathrm{a}}$ & $2.39^{\mathrm{a}}$ & $2.52^{\mathrm{a}}$ & $0.40^{\mathrm{a}}$ & $0.48^{\mathrm{a}}$ & $0.82^{\mathrm{a}}$ & $2.02^{\mathrm{a}}$ & $0.11^{\mathrm{a}}$ & $0.30^{\mathrm{a}}$ & $0.00^{\mathrm{a}}$ & $0.00^{\mathrm{b}}$ & $0.00^{\mathrm{a}}$ & $0.00^{\mathrm{a}}$ \\
\hline 15 & $1.02^{\mathrm{a}}$ & $1.45^{\mathrm{a}}$ & $0.22^{\mathrm{a}}$ & $0.25^{\mathrm{a}}$ & $1.64^{\mathrm{a}}$ & $1.97^{\mathrm{a}}$ & $0.25^{\mathrm{a}}$ & $0.39^{\mathrm{a}}$ & $1.13^{\mathrm{a}}$ & $0.47^{\mathrm{a}}$ & $0.18^{\mathrm{a}}$ & $0.14^{\mathrm{a}}$ & $0.00^{\mathrm{a}}$ & $0.00^{\mathrm{a}}$ & $0.00^{\mathrm{a}}$ & $0.00^{\mathrm{a}}$ \\
\hline
\end{tabular}

Means in the same column having the same letter(s) superscript are not significantly different at $\mathrm{P}<0.05$. WAP=Weeks after planting; FW=Fresh weight DW=Dry weight. 
Table 2: Effects of Organic (Cow dung and poultry litter) and Inorganic (NPK and Urea) fertilizers on leaf number of NH-Ae 47-4

\begin{tabular}{|c|c|c|c|c|c|c|c|c|c|c|c|c|c|c|c|c|c|c|c|c|c|c|c|c|}
\hline \multirow{2}{*}{ TREATMENT } & \multicolumn{6}{|c|}{$\begin{array}{c}\text { Cow dung } \\
\text { Week After Planting (WAP) }\end{array}$} & \multicolumn{6}{|c|}{$\begin{array}{c}\text { Poultry Litter } \\
\text { Week After Planting (WAP) }\end{array}$} & \multicolumn{6}{|c|}{$\begin{array}{c}\text { NPK } \\
\text { Week After Planting (WAP) }\end{array}$} & \multicolumn{6}{|c|}{$\begin{array}{c}\text { Urea } \\
\text { Week After Planting (WAP) }\end{array}$} \\
\hline & 3 & 4 & 5 & 6 & 7 & 8 & 3 & 4 & 5 & 6 & 7 & 8 & 3 & 4 & 5 & 6 & 7 & 8 & 3 & 4 & 5 & 6 & 7 & 8 \\
\hline Control & 6 & $5.00^{b}$ & $5.67^{\mathrm{a}}$ & $6.00 \mathrm{a}$ & $6.33^{\mathrm{a}}$ & $6.63^{\mathrm{a}}$ & $4.67^{\mathrm{a}}$ & $5.67^{\mathrm{a}}$ & $5.06^{\mathrm{a}}$ & $5.00^{\mathrm{a}}$ & $5.00^{a}$ & $5.00^{\mathrm{a}}$ & $4.67^{\mathrm{a}}$ & $5.33^{a}$ & $5.33^{\mathrm{a}}$ & $5.33^{\mathrm{a}}$ & $5.67^{\mathrm{a}}$ & $5.67^{\mathrm{a}}$ & $4.00^{\mathrm{a}}$ & $5.00^{\mathrm{a}}$ & $5.00^{\mathrm{a}}$ & $4.33^{\mathrm{a}}$ & $4.67^{\mathrm{a}}$ & $4.67^{\mathrm{a}}$ \\
\hline 5 & $4.67^{\mathrm{a}}$ & $5.33^{a}$ & $6.33^{\mathrm{a}}$ & $7.33^{\mathrm{a}}$ & $7.33^{\mathrm{a}}$ & $7.33^{\mathrm{a}}$ & $4.33^{\mathrm{a}}$ & $4.67^{\mathrm{a}}$ & $4.67^{\mathrm{a}}$ & $6.33^{\mathrm{a}}$ & $6.33^{\mathrm{a}}$ & $6.33^{\mathrm{a}}$ & $5.00^{\mathrm{a}}$ & $6.33^{a}$ & $4.67^{\mathrm{a}}$ & $4.67^{\mathrm{a}}$ & $4.67^{\mathrm{a}}$ & $5.33^{\mathrm{a}}$ & $3.33^{\mathrm{a}}$ & $3.67^{\mathrm{a}}$ & $3.33^{\mathrm{a}}$ & $2.67^{\mathrm{a}}$ & $2.67^{\mathrm{a}}$ & $3.00^{\mathrm{ab}}$ \\
\hline 10 & $4.67^{\mathrm{a}}$ & $5.33^{a}$ & $6.00^{\mathrm{a}}$ & $6.67^{a}$ & $7.33^{\mathrm{a}}$ & $7.33^{\mathrm{a}}$ & $5.00^{\mathrm{a}}$ & $5.33^{a}$ & $6.00^{\mathrm{a}}$ & $6.00^{\mathrm{a}}$ & $6.33^{\mathrm{a}}$ & $5.33^{\mathrm{a}}$ & $5.00^{\mathrm{a}}$ & $6.33^{a}$ & $4.67^{\mathrm{a}}$ & $3.67^{\mathrm{a}}$ & $3.67^{\mathrm{a}}$ & $3.67^{\mathrm{a}}$ & $0.67^{\mathrm{b}}$ & $0.00^{b}$ & $0.00^{\mathrm{b}}$ & $0.00^{\mathrm{a}}$ & $0.00^{\mathrm{a}}$ & $0.00^{\mathrm{b}}$ \\
\hline 15 & $4.67^{\mathrm{a}}$ & 5.67 & $6.33^{\mathrm{a}}$ & $6.00^{\mathrm{a}}$ & $7.00^{\mathrm{a}}$ & $8.00^{\mathrm{a}}$ & $5.00^{\mathrm{a}}$ & $6.00^{\mathrm{a}}$ & $6.00^{\mathrm{a}}$ & $7.00^{\mathrm{a}}$ & $7.33^{\mathrm{a}}$ & $7.00^{\mathrm{a}}$ & $4.33^{\mathrm{a}}$ & $5.33^{a}$ & $3.67^{\mathrm{a}}$ & $3.33^{\mathrm{a}}$ & $3.33^{\mathrm{a}}$ & $3.67^{\mathrm{a}}$ & $0.00^{b}$ & $6.00^{\mathrm{b}}$ & $0.00^{\mathrm{b}}$ & $0.00^{\mathrm{a}}$ & $0.00^{\mathrm{a}}$ & $0.00^{\mathrm{b}}$ \\
\hline
\end{tabular}

Means in the same column having the same letter(s) superscript are not significantly different at $\mathrm{P}<0.05$ WAP=Weeks after planting.

Plants treated with organic fertilizer; poultry litters and cow dung recorded higher leaf number, though cow dung has the highest (8) but was not significantly different from those treated with poultry litters (7). The Urea-grown okra variety had the least number of leaves while the other treatments had similar effects on leaf number with $(15 \mathrm{~g})$ cow dung-grown plants having the highest number of leaves at $8 \mathrm{WAP}$ (Table 2$)$.

Table 3: Effects of Organic (Cow dung and poultry litter) and Inorganic (NPK and Urea) fertilizers on leaf area of NH-Ae 47-4 Okra Variety

\begin{tabular}{|c|c|c|c|c|c|c|c|c|c|c|c|c|c|c|c|c|c|c|c|c|c|c|c|c|}
\hline \multirow{2}{*}{ TREATMENT } & \multicolumn{6}{|c|}{$\begin{array}{c}\text { Cow dung } \\
\text { Week After Planting (WAP) } \\
\end{array}$} & \multicolumn{6}{|c|}{$\begin{array}{c}\text { Poultry litter } \\
\text { Week After Planting (WAP) }\end{array}$} & \multicolumn{6}{|c|}{$\begin{array}{c}\text { NPK } \\
\text { Week After Planting (WAP) } \\
\end{array}$} & \multicolumn{6}{|c|}{$\begin{array}{c}\text { Urea } \\
\text { Week After Planting (WAP) }\end{array}$} \\
\hline & 3 & 4 & 5 & 6 & 7 & 8 & 3 & 4 & 5 & 6 & 7 & 8 & 3 & 4 & 5 & 6 & 7 & 8 & 3 & 4 & 5 & 6 & 7 & 8 \\
\hline Control & $28.67^{\mathrm{a}}$ & $33.33^{\mathrm{a}}$ & $38.00^{\mathrm{a}}$ & $38.67 \mathrm{a}$ & $39.00^{\mathrm{a}}$ & $39.00^{\mathrm{a}}$ & $30.00^{\mathrm{a}}$ & $41.67^{\mathrm{a}}$ & $42.67^{\mathrm{a}}$ & $44.00^{\mathrm{a}}$ & $45.67^{\mathrm{a}}$ & $46.33^{\mathrm{a}}$ & $28.33^{\mathrm{a}}$ & $32.67^{\mathrm{b}}$ & $34.67^{\mathrm{a}}$ & $42.33^{\mathrm{a}}$ & $43.00^{\mathrm{a}}$ & $43.00^{\mathrm{a}}$ & $22.00^{\mathrm{a}}$ & $26.00^{\mathrm{ab}}$ & $27.33^{\mathrm{a}}$ & $35.67^{\mathrm{a}}$ & $37.00^{\mathrm{a}}$ & $37.00^{\mathrm{a}}$ \\
\hline 5 & $39.33^{\mathrm{a}}$ & $48.33^{\mathrm{a}}$ & $50.33^{\mathrm{a}}$ & $52.00^{\mathrm{a}}$ & $53.00^{\mathrm{a}}$ & $53.33^{\mathrm{a}}$ & $35.00^{\mathrm{a}}$ & $49.67^{\mathrm{a}}$ & $48.67^{\mathrm{a}}$ & $50.67^{\mathrm{a}}$ & $52.33^{\mathrm{a}}$ & $52.33^{\mathrm{a}}$ & $67.67^{\mathrm{a}}$ & $68.00^{\mathrm{a}}$ & $52.67^{\mathrm{a}}$ & $55.67^{\mathrm{a}}$ & $56.67^{\mathrm{a}}$ & $56.67^{\mathrm{a}}$ & $19.67^{\mathrm{a}}$ & $28.33^{\mathrm{a}}$ & $32.67^{\mathrm{a}}$ & $26.33^{\mathrm{a}}$ & $26.67^{\mathrm{a}}$ & $26.67^{\mathrm{a}}$ \\
\hline 10 & $39.33^{\mathrm{a}}$ & $46.67^{\mathrm{a}}$ & $48.00^{\mathrm{a}}$ & $49.00^{\mathrm{a}}$ & $50.00^{\mathrm{a}}$ & $50.33^{\mathrm{a}}$ & $49.33^{\mathrm{a}}$ & $57.00^{\mathrm{a}}$ & $58.00^{\mathrm{a}}$ & $61.33^{\mathrm{a}}$ & $62.67^{\mathrm{a}}$ & $62.33^{\mathrm{a}}$ & $52.67^{\mathrm{a}}$ & $83.00^{\mathrm{a}}$ & $40.00^{\mathrm{a}}$ & $34.67^{\mathrm{a}}$ & $35.67^{\mathrm{a}}$ & $35.67^{\mathrm{a}}$ & $1.33^{\mathrm{b}}$ & $0.00^{b}$ & $0.00^{\mathrm{a}}$ & $0.00^{\mathrm{a}}$ & $0.00^{\mathrm{a}}$ & $0.00^{\mathrm{a}}$ \\
\hline 15 & $38.67^{\mathrm{a}}$ & $53.67^{\mathrm{a}}$ & $52.33^{\mathrm{a}}$ & $52.00^{\mathrm{a}}$ & $57.67^{\mathrm{a}}$ & $54.33^{\mathrm{a}}$ & $48.00^{\mathrm{a}}$ & $66.33^{\mathrm{a}}$ & $62.67^{\mathrm{a}}$ & $63.33^{\mathrm{a}}$ & $64.33^{\mathrm{a}}$ & $64.67^{\mathrm{a}}$ & $24.33^{\mathrm{a}}$ & $49.67^{\mathrm{a}}$ & $29.67^{\mathrm{a}}$ & $41.67^{\mathrm{a}}$ & $42.00^{\mathrm{a}}$ & $42.00^{\mathrm{a}}$ & $0.00^{\mathrm{b}}$ & $0.00^{\mathrm{b}}$ & $0.00^{\mathrm{a}}$ & $0.00^{\mathrm{a}}$ & $0.00^{\mathrm{a}}$ & $0.00^{\mathrm{a}}$ \\
\hline
\end{tabular}

Means in the same column having the same letter(s) superscript are not significantly different at $\mathrm{P}<0.05$.

WAP=Weeks after planting. 
Poultry litter $(10 \mathrm{~g}$ and $15 \mathrm{~g})$ treated okra variety had the highest leaf area than in cow dung of same concentration throughout the weeks of planting. However, okra variety planted in $5 \mathrm{~g} \mathrm{NPK}$ treated soil had the highest leaf area than other treatments with the same concentration while okra variety in Urea treated soil has the lowest (Table 3).

Table 4: Effects of Organic (Cow dung and poultry litter) and Inorganic (NPK and Urea) fertilizers on stem height (cm) of NH-Ae 47-4

\begin{tabular}{|c|c|c|c|c|c|c|c|c|c|c|c|c|c|c|c|c|c|c|c|c|c|c|c|c|}
\hline \multirow{2}{*}{ TREATMENT } & \multicolumn{6}{|c|}{$\begin{array}{c}\text { Cow dung } \\
\text { Week After Planting (WAP) }\end{array}$} & \multicolumn{6}{|c|}{$\begin{array}{c}\text { Poultry litter } \\
\text { Week After Planting (WAP) }\end{array}$} & \multicolumn{6}{|c|}{$\begin{array}{c}\text { NPK } \\
\text { Week After Planting (WAP) }\end{array}$} & \multicolumn{6}{|c|}{$\begin{array}{c}\text { Urea } \\
\text { Week After Planting (WAP) }\end{array}$} \\
\hline & 3 & 4 & 5 & 6 & 7 & 8 & 3 & 4 & 5 & 6 & 7 & 8 & 3 & 4 & 5 & 6 & 7 & 8 & 3 & 4 & 5 & 6 & 7 & 8 \\
\hline Control & $9.00^{\mathrm{a}}$ & $13.07^{\mathrm{a}}$ & $16.67^{\mathrm{a}}$ & $17.33^{\mathrm{a}}$ & $17.67^{\mathrm{a}}$ & $18.33^{\mathrm{a}}$ & $7.83^{\mathrm{a}}$ & $14.40^{\mathrm{a}}$ & $18.50^{\mathrm{a}}$ & $19.67^{\mathrm{a}}$ & $20.33^{\mathrm{a}}$ & $20.33^{\mathrm{a}}$ & $7.34^{\mathrm{a}}$ & $13.57^{\mathrm{a}}$ & $18.00^{\mathrm{a}}$ & $19.00^{\mathrm{a}}$ & $19.33^{\mathrm{a}}$ & $20.33^{\mathrm{a}}$ & $5.33^{\mathrm{a}}$ & $13.00^{\mathrm{a}}$ & $14.33^{\mathrm{a}}$ & $15.00^{\mathrm{a}}$ & $15.67^{\mathrm{a}}$ & $16.00^{\mathrm{a}}$ \\
\hline 5 & $9.43^{\mathrm{a}}$ & $15.17^{\mathrm{a}}$ & $20.00^{\mathrm{a}}$ & $21.33^{\mathrm{ab}}$ & $22.67^{\mathrm{ab}}$ & $23.67^{\mathrm{ab}}$ & $8.83^{\mathrm{a}}$ & $14.77^{\mathrm{a}}$ & $18.33^{\mathrm{a}}$ & $19.67^{\mathrm{a}}$ & $20.67^{\mathrm{a}}$ & $21.00^{\mathrm{a}}$ & $10.17^{\mathrm{a}}$ & $16.23^{\mathrm{a}}$ & $16.50^{\mathrm{a}}$ & $17.67^{\mathrm{a}}$ & $18.33^{\mathrm{a}}$ & $18.33^{\mathrm{a}}$ & $5.83^{\mathrm{a}}$ & $16.23^{\mathrm{a}}$ & $11.67^{\mathrm{a}}$ & $7.33^{\mathrm{ab}}$ & $7.67^{\mathrm{ab}}$ & $8.00^{\mathrm{ab}}$ \\
\hline 10 & $10.00^{\mathrm{a}}$ & $16.17^{\mathrm{a}}$ & $19.00^{\mathrm{a}}$ & $21.33^{\mathrm{ab}}$ & $22.67^{\mathrm{ab}}$ & $23.00^{\mathrm{ab}}$ & $8.00^{\mathrm{a}}$ & $15.70^{\mathrm{b}}$ & $20.67^{\mathrm{a}}$ & $23.33^{\mathrm{a}}$ & $23.33^{\mathrm{a}}$ & $24.00^{\mathrm{a}}$ & $5.83^{\mathrm{b}}$ & $12.27^{\mathrm{a}}$ & $11.00^{\mathrm{a}}$ & $11.33^{\mathrm{a}}$ & $11.67^{\mathrm{a}}$ & $11.67^{\mathrm{a}}$ & $0.67^{\mathrm{b}}$ & $0.00^{\mathrm{b}}$ & $0.00^{\mathrm{b}}$ & $0.00^{\mathrm{b}}$ & $0.00^{\mathrm{b}}$ & $0.00^{\mathrm{b}}$ \\
\hline 15 & $9.00^{\mathrm{a}}$ & $18.33^{\mathrm{a}}$ & $22.67^{\mathrm{a}}$ & $26.67^{\mathrm{b}}$ & $28.00^{\mathrm{b}}$ & $28.67^{\mathrm{ab}}$ & $9.33^{\mathrm{b}}$ & $16.43^{b}$ & $22.67^{\mathrm{a}}$ & $29.00^{\mathrm{a}}$ & $29.00^{\mathrm{a}}$ & $29.33^{\mathrm{a}}$ & $4.43^{\mathrm{b}}$ & $8.33^{\mathrm{a}}$ & $7.33^{\mathrm{a}}$ & $8.33^{\mathrm{a}}$ & $8.67^{\mathrm{a}}$ & $9.67^{\mathrm{a}}$ & $0.00^{\mathrm{b}}$ & $0.00^{\mathrm{b}}$ & $0.00^{\mathrm{b}}$ & $0.00^{\mathrm{b}}$ & $0.00^{\mathrm{b}}$ & $0.00^{\mathrm{b}}$ \\
\hline
\end{tabular}

Means in the same column having the same letter(s) superscript are not significantly different at $\mathrm{P}<0.05$.

WAP=Weeks after planting.

Poultry litters treated plants have highest stem height across the concentrations though it is not significantly different from its control and those treated with NPK

(Table 4).

Table 5: Effects of Organic (Cow dung and poultry litter) and Inorganic (NPK and Urea) fertilizers on stem girth of NH-Ae 47-4

\begin{tabular}{|c|c|c|c|c|c|c|c|c|c|c|c|c|c|c|c|c|c|c|c|c|c|c|c|c|}
\hline \multirow[t]{2}{*}{ TREATMENT } & \multicolumn{6}{|c|}{$\begin{array}{c}\text { Cow dung } \\
\text { Week After Planting (WAP) }\end{array}$} & \multicolumn{6}{|c|}{$\begin{array}{c}\text { Poultry litter } \\
\text { Week After Planting (WAP) }\end{array}$} & \multicolumn{6}{|c|}{$\begin{array}{c}\text { NPK } \\
\text { Week After Planting (WAP) }\end{array}$} & \multicolumn{6}{|c|}{$\begin{array}{c}\text { Urea } \\
\text { Week After Planting (WAP) }\end{array}$} \\
\hline & 3 & 4 & 5 & 6 & 7 & 8 & 3 & 4 & 5 & 6 & 7 & 8 & 3 & 4 & 5 & 6 & 7 & 8 & 3 & 4 & 5 & 6 & 7 & 8 \\
\hline Control & $1.20^{\mathrm{a}}$ & $1.47^{\mathrm{a}}$ & $1.50^{\mathrm{a}}$ & $1.53^{\mathrm{a}}$ & $1.60^{\mathrm{a}}$ & $1.60^{\mathrm{a}}$ & $1.37^{\mathrm{a}}$ & $1.53^{\mathrm{a}}$ & $1.53^{\mathrm{a}}$ & $1.60^{\mathrm{a}}$ & $1.60^{\mathrm{a}}$ & $1.63^{\mathrm{a}}$ & $1.33^{\text {ab }}$ & $1.80^{\mathrm{a}}$ & $1.50^{\mathrm{a}}$ & $1.53^{\mathrm{a}}$ & $1.53^{\mathrm{a}}$ & $1.53^{\mathrm{a}}$ & $1.43^{\mathrm{a}}$ & $1.60^{\mathrm{a}}$ & $1.63^{\mathrm{a}}$ & $1.63^{\mathrm{a}}$ & $1.63^{\mathrm{a}}$ & $1.67^{\mathrm{a}}$ \\
\hline 5 & $1.40^{\mathrm{a}}$ & $1.63^{\mathrm{a}}$ & $1.60^{\mathrm{a}}$ & $1.67^{\mathrm{a}}$ & $1.77^{\mathrm{a}}$ & $1.77^{\mathrm{a}}$ & $1.40^{\mathrm{a}}$ & $1.67^{\mathrm{a}}$ & $1.73^{\mathrm{a}}$ & $1.77^{\mathrm{a}}$ & $1.80^{\mathrm{a}}$ & $1.83^{\mathrm{a}}$ & $1.93^{\mathrm{b}}$ & $1.83^{\mathrm{a}}$ & $1.40^{\mathrm{a}}$ & $1.43^{\mathrm{a}}$ & $1.43^{\mathrm{a}}$ & $1.43^{\mathrm{a}}$ & $1.03^{\mathrm{a}}$ & $1.17^{\mathrm{a}}$ & $1.43^{\mathrm{a}}$ & $0.53^{\mathrm{b}}$ & $0.53^{\mathrm{b}}$ & $0.53^{\mathrm{b}}$ \\
\hline 10 & $1.50^{\mathrm{a}}$ & $1.70^{\mathrm{a}}$ & $1.73^{\mathrm{a}}$ & $1.77^{\mathrm{a}}$ & $1.80^{\mathrm{a}}$ & $1.80^{\mathrm{a}}$ & $1.67^{\mathrm{a}}$ & $1.83^{\mathrm{a}}$ & $1.90^{\mathrm{a}}$ & $1.93^{\mathrm{a}}$ & $1.93^{\mathrm{a}}$ & $1.93^{\mathrm{a}}$ & $1.70^{\mathrm{ab}}$ & $1.87^{\mathrm{a}}$ & $1.03^{\mathrm{a}}$ & $1.03^{\mathrm{a}}$ & $1.07^{\mathrm{a}}$ & $1.10^{\mathrm{a}}$ & $0.23^{\mathrm{b}}$ & $0.00^{\mathrm{b}}$ & $0.00^{\mathrm{b}}$ & $0.00^{\mathrm{b}}$ & $0.00^{\mathrm{b}}$ & $0.00^{\mathrm{b}}$ \\
\hline 15 & $1.46^{\mathrm{a}}$ & $1.73^{\mathrm{a}}$ & $1.73^{\mathrm{a}}$ & $1.74^{\mathrm{a}}$ & $1.83^{\mathrm{a}}$ & $1.83^{\mathrm{a}}$ & $1.77^{\mathrm{a}}$ & $1.97^{\mathrm{a}}$ & $1.97^{\mathrm{a}}$ & $2.03^{\mathrm{a}}$ & $2.10^{\mathrm{a}}$ & $2.23^{\mathrm{a}}$ & $0.87^{\mathrm{a}}$ & $1.13^{\mathrm{a}}$ & $0.77^{\mathrm{a}}$ & $0.83^{\mathrm{a}}$ & $0.83^{\mathrm{a}}$ & $0.83^{\mathrm{a}}$ & $0.00^{\mathrm{b}}$ & $0.00^{\mathrm{b}}$ & $0.00^{b}$ & $0.00^{\mathrm{b}}$ & $0.00^{\mathrm{b}}$ & $0.00^{\mathrm{b}}$ \\
\hline
\end{tabular}


Means in the same column having the same letter(s) superscript are not significantly different at $\mathrm{P}<0.05$.

WAP $=$ Weeks after planting.

Similar to results recorded for stem height of plants treated, plants treated with poultry litters also have the highest stem girth $(2.23 \mathrm{~cm} 0$ at 8 WAP. However, plants treated with cow dung showed higher stem girth when compared with those treated with NPK (Table 5).

Optimum crop performance is usually limited by inadequate availability of essential nutrients. The results of this research highlighted the superiority of fertilized plants over non-fertilized and poorly fertilized (urea) ones in term of growth. The consistently poor performance of non-fertilized plants shows that when nutrients are available in adequate amount there is tendency for plants to produce at their optimum potential. The experiment reveals that NPK fertilizer; poultry litter and cow dung application significantly influenced the growth of the NH-Ae 47-4 variety of okra. The higher performance of crops in NPK over poultry litter and cow dung at 3 and 4 WAP is indicative of the ease of dissolution of nutrients in the inorganic fertilizer being in a more soluble form while the organic manure was released slowly. This is in accordance with Ayeni et al. (2012) and who stated that poultry litter and cow dung also contains useful soil nutrients that are needed for the growth of plants, but their composition is in the crude form that is released slowly to the soil.

The fresh and dry shoot weights were affected by fertilizer application, NPK fertilizer had the highest fresh and dry shoot weights at 3 WAP than other treatments but poultry litter (10 and 15 grams) had the overall highest fresh and dry shoot weight in the NH-Ae 47-4 variety of okra planted. This is accordance with Oladotun (2002) who reported that poultry manure contains a relatively high nitrogen content that supports vegetative growth of crops. The results are also in agreement with those reported by Massomo and Rweyemamu (1989) who observed significant effects on yield of common beans following application of poultry manure.

Leaf number was highest with cow dung treated plants of $\mathrm{NH}$ Ae 47-4 variety of okra. Other treatments have similar effects on leaf number with urea treated plants having the least number of leaves. This was in accordance with Tindall (1992) who reported that the increase in number of leaves per plant with organic fertilizer application stressed its importance during the vegetative growth of crop plants.
Leaf area was highest in NPK treated plants of the NH Ae 47-4 variety when it was applied in low concentration but poultry litter had the overall highest leaf area which is in accordance with the findings of Oladotun (2002) who reported similar thing in okra.

Stem height and stem girth was initially higher in NPK and Urea treated plants of the NH Ae 47-4 variety of okra, but dropped and was lower than other treatments, poultry litter treated plants having overall highest stem height and stem girth. This was in accordance with Ajari et al. (2003) in which they reported that organic manure especially poultry manure could increase plant height when compared with other sources of manure.

Conclusion: The application of poultry litter, cow dung, NPK fertilizer and urea fertilizer had a significant effect on vegetative growth of $\mathrm{NH}$ Ae 47-4 variety of okra. The results obtained revealed that this variety responded well to the application of organic manure compared to inorganic fertilizer and control treatment in the study. Based on the findings of this study, it may be recommended that applying organic manure was adequate for maximum growth and yield of $\mathrm{NH} \mathrm{Ae}$ 47-4 variety of okra and 
inorganic fertilizers especially NPK fertilizer may be applied in the absence of organic manure.

\section{REFERENCES}

Aladele, S.E., Ariyo, O.J. and Lapena, R. (2008). Genetic Relationship among West African Okra (Abelmoschus caillei) and Asian genotypes (Abelmoschus esculentus) using RAPD. African Journal Biotechnology, 7: 1426-143.

Ajari, O., Tsado, L. E. K., Oladiran, J. A. and Salako, E. A. (2003). Plant height and fruit yield of okra as affected by field application of fertilizer and organic matter in Bida, Nigeria. The Nigerian Agricultural Journal, 34: 74 - 80.

Ayeni, L. S., Adeleye, E, O. and Adejumo, J. O. (2012). Comparative effect of organic, organomineral and mineral fertilizers on soil properties, nutrient uptake, growth and yield of Maize (Zea mays). International Research Journal of Agricultural Science and Soil Science, 2(11):493-497.

Brady, N.C. and Weil, R. (1999). The Nature and Properties of soil. 12th Edition. Mac. Pub. Com. New York. 625-640.

Chauhan, D. V. S. (1972). Vegetable Production in India (3rd edition). Ram Prasad and Sons, Agra.

Hedrick, U. P. (1972). Sturtevant's Edible Pl ants of the World. Dover Publications.

Hera, C. (1996). Role of Inorganic fertilizer and their management practices. Kluwer Academic Publishers Fert. Res. 43:63-81.
Massomo, S. M. S. and Rweyemamu, C. L. (1989). Evaluation of the effects of cattle and poultry manure in combination with inorganic $\mathrm{N}$ fertilizer on seed yield, yield components and seed quality of common bean ( $P$. vulgaris L.) grown in different plant stands per hill. In: Bean Research. Maeda and Nchimbi (Eds.). 4: 88-98.

Oladotun, A.O., (2002). Managing manure as a fertilizer; Saskatchewani, Agriculture, Food and Rural Revitalization, pp. 5.

Qhureshi, Z. (2007). Breeding Investigation in Bhendi (Abelmoschus esculentus (L.) Moench). Master Thesis, University of Agriculture Sciences, GKVK, Bangalore.

Sanchaz, P. A., Shapher, K. D., Soul, M. J., Place, E. M., Momwunyea, A. U., Buresh, R. J., Kwesiga, F.R., Izac, A. M., Nderitu, C. G. and Woomer, P. L. (1997). Soil fertility replenishment in Africa: an investment in natural resource capital in: replenishing soil fertility in Africa,Eds.

Shukla, V.and Nalk, L. B. (1993). AgroTechnique for Malvaceae Vegetables, In: Ifovance in horticulture vol 5. Malhotra publishing house, New Delhi, India, pp 399. 425.

Tindall, H. D. and Rice, R. P. (1983). Fruit and Vegetable Production in Warm Climates. The Macmillan press Ltd Nigeria. 85 pp.

Tindall, H.D. (1992). Vegetables in the Tropics. Macmillan Press Ltd, London, pp. 533.

Varmudy, V. (2011). Marking survey need to boost okra exports. Department of economics, Vivekananda College, Puttur, Karnataka, India. 